\title{
Safer In Vitro Drug Screening Models for Melioidosis Therapy Development
}

\author{
Anna S. Amiss, ${ }^{1}$ Jessica R. Webb, ${ }^{2}$ Mark Mayo, ${ }^{2}$ Bart J. Currie, ${ }^{2,3}$ David J. Craik, ${ }^{1}$ Sónia Troeira Henriques,,${ }^{1,4 \star}$ \\ and Nicole Lawrence ${ }^{1 *}$ \\ ${ }^{1}$ Institute for Molecular Bioscience, The University of Queensland, Brisbane, Australia; ${ }^{2}$ Global and Tropical Health Division, Menzies School of \\ Health Research, Darwin, Australia; ${ }^{3}$ Northern Territory Medical Program, Department of Infectious Diseases, Royal Darwin Hospital, Darwin, \\ Australia; ${ }^{4}$ Queensland University of Technology, School of Biomedical Sciences, Institute of Healthy and Biomedical Innovation, and \\ Translational Research Institute, Brisbane, Queensland, Australia
}

\begin{abstract}
Melioidosis is a neglected tropical disease caused by the Gram-negative soil bacterium Burkholderia pseudomallei. Current antibiotic regimens used to treat melioidosis are prolonged and expensive, and often ineffective because of intrinsic and acquired antimicrobial resistance. Efforts to develop new treatments for melioidosis are limited by the risks associated with handling pathogenic $B$. pseudomallei, which restricts research to facilities with biosafety level three containment. Closely related nonpathogenic Burkholderia can be investigated under less stringent biosafety level two containment, and we hypothesized that they could be used as model organisms for developing therapies that would also be effective against $B$. pseudomallei. We used microbroth dilution assays to compare drug susceptibility profiles of three B. pseudomallei strains and five nonpathogenic Burkholderia strains. Burkholderia humptydooensis, Burkholderia thailandensis, and Burkholderia territorii had similar susceptibility profiles to pathogenic $B$. pseudomallei that support their potential as safer in vitro models for developing new melioidosis therapies.
\end{abstract}

\section{INTRODUCTION}

Burkholderia pseudomallei is a Gram-negative bacterium that causes melioidosis, ${ }^{1,2}$ a neglected tropical disease, with one model estimating up to 165,000 cases and 89,000 deaths per year. ${ }^{1}$ Mortality rates for infected individuals vary from under $10 \%$ in Darwin (Northern Territory, Australia), ${ }^{3}$ where state-of-the-art intensive care facilities are available, to more than $40 \%$ in endemic regions in Southeast Asia, where health resources are more limited. ${ }^{4}$

Conventional large-spectrum antibiotic classes, such as aminoglycosides (e.g., streptomycin, gentamicin, and kanamycin), early generation $\beta$-lactam antibiotics (e.g., penicillin), fluoroquinolones (e.g., ciprofloxacin), and macrolides (e.g., erythromycin), have little effect on $B$. pseudomallei, which limits treatment options. ${ }^{5-8}$ Importantly, environmental isolates and primary $B$. pseudomallei isolates are almost universally susceptible to the first-line drugs used for melioidosis therapy, ceftazidime, meropenem, and co-trimoxazole. ${ }^{5,9,10}$ However, the prolonged nature of melioidosis treatment (46 months) can lead to acquired antibiotic resistance, which is linked to treatment failure and mortality. ${ }^{9}$ To overcome both intrinsic and acquired antibiotic resistance, more efficacious therapies for melioidosis are required. ${ }^{1,11}$

Efforts to develop new treatments for melioidosis are limited by the classification of $B$. pseudomallei as a risk group three microorganism (i.e., the potential to cause serious human disease) in most countries. ${ }^{12-15}$ This classification restricts its research to laboratories classified as biosafety level three in the United States, ${ }^{16}$ or the equivalent physical containment three in Australia and New Zealand. ${ }^{17}$ Burkholderia pseudomallei is also recognized as a tier one biothreat agent on the

* Address correspondence to Nicole Lawrence, Institute for Molecular Bioscience, The University of Queensland, 306 Carmody Rd., Brisbane 4072, Australia, E-mail: n.lawrence@uq.edu.au or Sónia Troeira Henriques, Queensland University of Technology, School of Biomedical Sciences, Institute of Healthy and Biomedical Innovation, and Translational Research Institute, Brisbane, Queensland, 4102, Australia, E-mail: sonia.henriques@qut.edu.au.
Center for Disease Control and Prevention Bioterrorism Agent list, ${ }^{15}$ a classification that further restricts research efforts. ${ }^{18,19}$

The use of nonpathogenic Burkholderia species as models for $B$. pseudomallei is a desirable approach for overcoming these limitations. Burkholderia thailandensis ${ }^{20-22}$ and mutant B. pseudomallei strains Bp82 and Bp190 that are avirulent to mice and hamsters ${ }^{23}$ have been used as model organisms because of their close genetic relationship to $B$. pseudomallei, but extensive comparison of their susceptibility to antibiotics or other therapeutic compounds has not yet been described. In addition to these established models, we propose that other closely related Burkholderia species that are not implicated in human disease $\mathrm{e}^{24-26}$ will also be useful model organisms for melioidosis research.

To determine their suitability as model organisms, we characterized the antibiotic susceptibility of five nonpathogenic Burkholderia strains and three B. pseudomallei strains to a panel of antibiotics and drug-like compounds. Here, we demonstrate that B. thailandensis and two newly characterized strains-Burkholderia humptydooensis and Burkholderia territorii-have susceptibility profiles that recapitulate $B$. pseudomallei susceptibility. The similarity of these profiles supports the utility of nonpathogenic Burkholderia as models for initial melioidosis therapy development.

\section{MATERIALS AND METHODS}

Burkholderia strains. Burkholderia strains used in this study were B. pseudomallei (MSHR10517, MSHR2154, and MSHR1364), B. humptydooensis (MSMB043), Burkholderia oklahomensis (MSMB0175), Burkholderia stagnalis (MSMB049), B. thailandensis (MSMB0608), and B. territorii (MSMB0110). These strains were collected and cultivated from soil or water samples as previously described (Menzies School of Health Research). ${ }^{27-29}$

Antibiotic panel. Antibiotics that have been included in this study represent the current standard therapeutics for treating melioidosis, ceftazidime, co-trimoxazole, and meropenem ${ }^{5}$; and other antibiotics used to treat bacterial infections. To produce a wider susceptibility profile, antibiotics with varying levels of efficacy against $B$. pseudomallei $i^{7,30-36}$ were also included (see Table 1). 


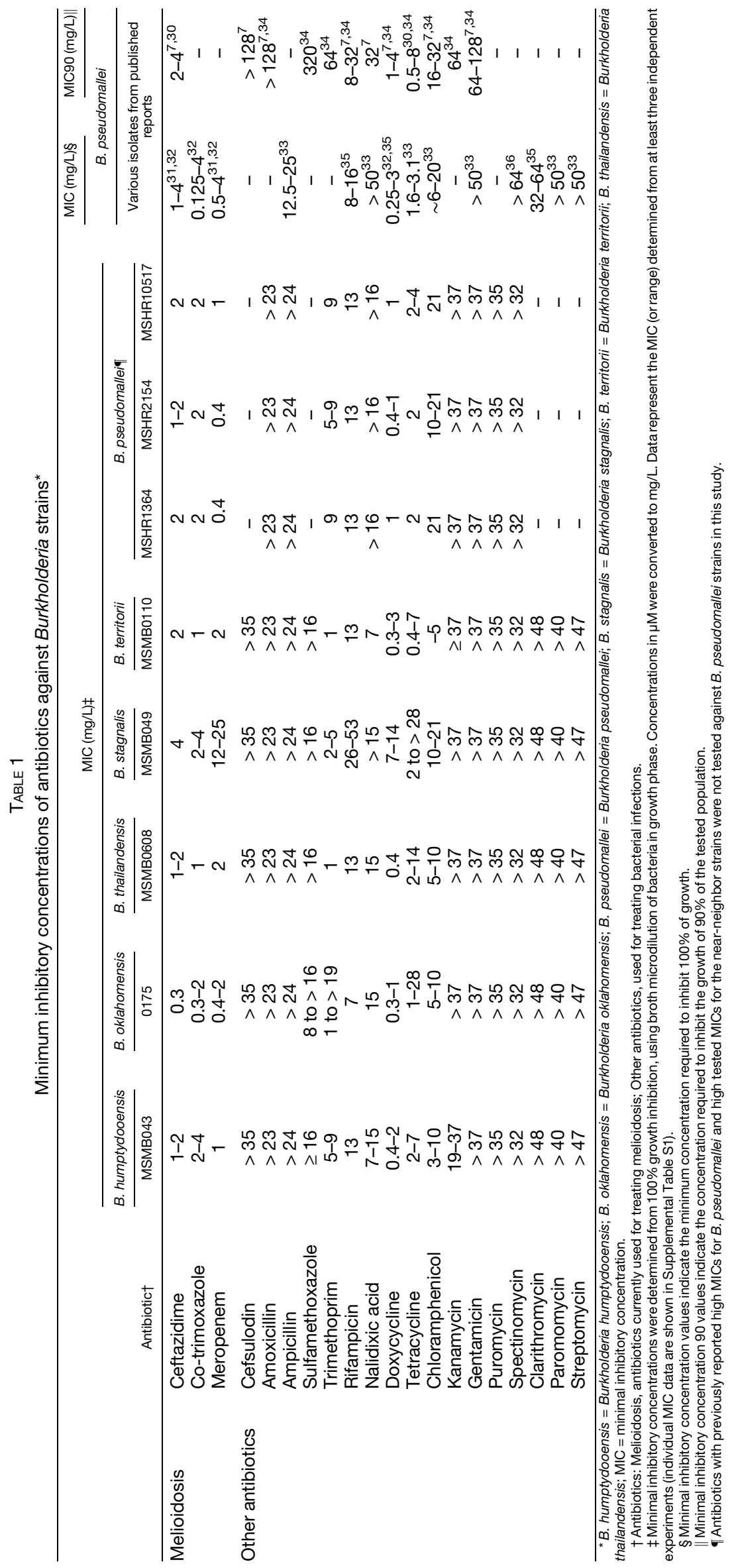




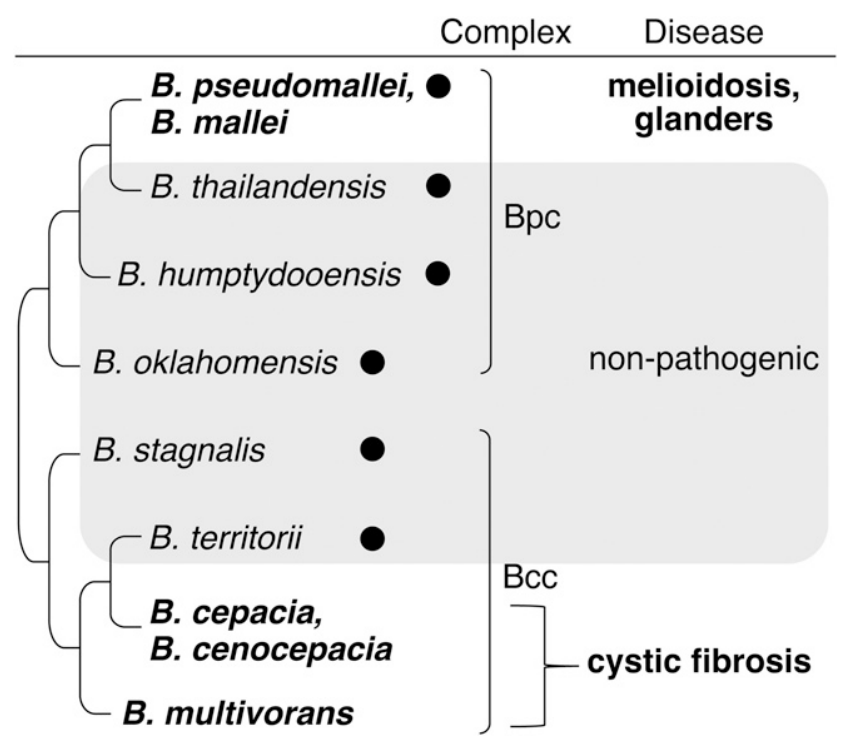

FIGURE 1. Schematic representation of near-neighbor Burkholderia species and their relatedness to Burkholderia pseudomallei and other major disease-causing species. Relationships are derived from previous phylogenetic trees. ${ }^{24-26,43}$ Lines represent relationships between the species but not genetic distance. Closed circles indicate Burkholderia species included in this study. The species highlighted in bold are implicated in human disease.

Antibiotic susceptibility profiles. Antimicrobial susceptibility was tested using a plate-based broth microdilution method as before, ${ }^{37}$ except that bacteria were incubated at $30^{\circ} \mathrm{C}$ because they showed better growth at $30^{\circ} \mathrm{C}$ than at $25^{\circ} \mathrm{C}$ and $37^{\circ} \mathrm{C}$. In brief, assays were conducted in Mueller Hinton broth with bacteria in mid-log-phase growth that were diluted to $\sim 10^{6}$ colony-forming unit (CFU)/mL (optical density at 600 $\mathrm{nm}=0.001$ ). Serial dilutions of the compounds were then added to the bacteria (final density $\sim 5 \times 10^{5} \mathrm{CFU} / \mathrm{mL}$ ). The minimal inhibitory concentration (MIC) was determined to be the lowest concentration of compound that inhibited visible bacterial growth 24 hours after treatment. Data represent the MIC determined from a minimum of three independent experiments for each strain (see Supplemental Table S1 for individual MIC data for all replicates). Principal component analyses were performed using the "prcomp" function, and plots were generated using the "ggplot" function of R version $4.0 .0 .^{38}$
Medicines for Malaria Venture (MMV) Pathogen Box compound susceptibility profiles for $B$. humptydooensis and $\boldsymbol{B}$. territorii. We tested the antimicrobial activity of the MMV Pathogen Box containing a library of 400 drug-like compounds. ${ }^{39}$ Initial antimicrobial susceptibility testing was performed at $20 \mu \mathrm{M}$ against $B$. humptydooensis and $B$. territorii, using the methods described earlier. Ceftazidime $(20 \mu \mathrm{M})$ was added to each plate as a positive control for $100 \%$ growth inhibition. ${ }^{5}$ We identified a subset of compounds with promising activity (compound ID, molecular weight, molecular formula, and structure of compounds from this subset are provided in Supplemental Table S2). These compounds were serially diluted to determine the MIC toward $B$. humptydooensis and $B$. territorii. Data represent the MIC determined from three independent experiments for each strain.

\section{RESULTS AND DISCUSSION}

Burkholderia near-neighbor strains. The Burkholderia genus comprises more than 70 species with varying virulence and pathogenicity, ${ }^{40-42}$ and these species can be divided according to their relationship to either B. pseudomallei (the B. pseudomallei complex) or Burkholderia cepacia (the B. cepacia complex). In the current study, we included three strains of $B$. pseudomallei and five representative near-neighbor Burkholderia strains of $B$. thailandensis, B. humptydooensis, B. oklahomensis, $B$. stagnalis, and $B$. territorii. The relationship between the nonpathogenic near neighbors and pathogenic Burkholderia species is represented in Figure 1. Genetic distance from B. pseudomallei is shown in Supplemental Table S3 for corresponding nearneighbor isolates from a previous study. ${ }^{43}$

Antibiotic susceptibility profiles for $B$. pseudomallei and near neighbors. The aim of this investigation was to determine whether the antibiotic susceptibility of $B$. pseudomallei is recapitulated by near-neighbor species. We hypothesize that less pathogenic species with similar antibiotic susceptibility profiles to $B$. pseudomallei are useful as in vitro models to facilitate initial screening of new therapeutic molecules without the restrictive physical containment requirements required when working with $B$. pseudomallei.

We determined the susceptibility of the B. pseudomallei and near-neighbor strains to a panel of 20 antibiotics with previously reported MIC90 or MIC values against B. pseudomallei. The MICs (or MIC range) are compared in
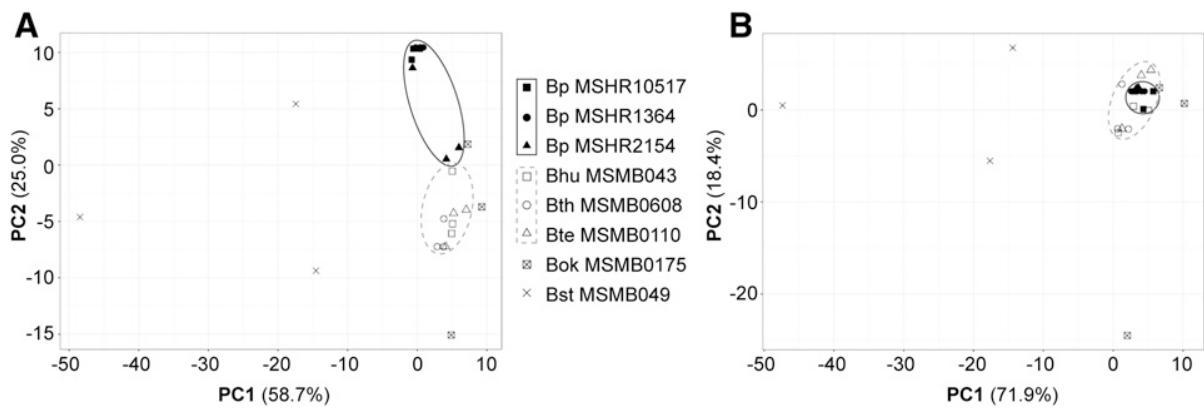

FIGURE 2. Principal component analysis ${ }^{38}$ of minimal inhibitory concentrations of antibiotics against Burkholderia strains: Burkholderia pseudomallei (Bp), Burkholderia humptydooensis (Bhu), Burkholderia thailandensis (Bth), Burkholderia territorii (Bte), Burkholderia oklahomensis (Bok), and Burkholderia stagnalis (Bst). (A) Analysis includes ceftazidime, co-trimoxazole, meropenem, trimethoprim, rifampicin, doxycycline, tetracycline, and chloramphenicol. (B) Exclusion of chloramphenicol from the analysis. The percentages of the total variance along the principal component 1 (PC1) and PC2 axes are shown in brackets. 
Table 1 and show a similar overall susceptibility profile for all of the tested strains. Principal component analysis (PCA) of MICs from the eight most potent antibiotics—ceftazidime, cotrimoxazole, meropenem, trimethoprim, rifampicin, doxycycline, tetracycline, and chloramphenicol-revealed that $B$. humptydooensis, B. thailandensis, and B. territorii display a similar susceptibility to these antibiotics as $B$. pseudomallei strains MSHR10517, MSHR2154, and MSHR1364 (Figure 2A). The MICs of chloramphenicol for these B. pseudomallei strains may be considered as outliers, as they are at the higher end of the reported MIC range for $B$. pseudomallei ( 6-20 mg/L). Indeed, removal of chloramphenicol MICs from the PCA analysis resulted in tighter clustering of $B$. humptydooensis, $B$. thailandensis, and $B$. territorii with $B$. pseudomallei (Figure 2B).

Minimal inhibitory concentrations for the antibiotics used to treat melioidosis were similar between near-neighbor $B$. humptydooensis, $B$. thailandensis, and B. territorii strains; $B$. pseudomallei strains from this study; and previously reported MICs for ceftazidime (1-2 mg/L), co-trimoxazole (2 mg/L), and meropenem (0.4-1 $\mathrm{mg} / \mathrm{L})$ against various $B$. pseudomallei strains (see Table 1). Minimal inhibitory concentrations for these antibiotics toward $B$. oklahomensis were slightly less predictive of $B$. pseudomallei susceptibility, and $B$. stagnalis MICs were even less predictive, especially for meropenem (MIC 12-25 mg/L).

The activity of other antibiotics used to treat bacterial infection further illustrated the utility of $B$. humptydooensis, $B$. thailandensis, and B. territorii as models for B. pseudomallei. Rifampicin had near-identical activity toward these nearneighbor strains and $B$. pseudomallei (MIC $\sim 13 \mathrm{mg}$, see Table 1). Minimal inhibitory concentration ranges for doxycycline were similar between the near neighbors and $B$. pseudomallei (0.3-3 versus $0.4-1 \mathrm{mg} / \mathrm{L}$ ), and the near neighbors were equally, or more, susceptible to trimethoprim (MIC 1-9 mg/L) and chloramphenicol (MIC 3-10 mg/L) than the B. pseudomallei strains (MIC 5-9 mg/L and 10-21 mg/L, respectively). Tetracycline susceptibility of the $B$. pseudomallei strains (MIC 2-4 mg/L) was within the range of MICs observed for $B$. humptydooensis, $B$. thailandensis, and $B$. territorii. The wider range of MICs observed for tetracycline (see Table 1, Supplemental Table S1) may reflect the bacteriostatic mechanism of action; however, this was not observed for other bacteriostatic antibiotics, including doxycycline and trimethoprim.
Kanamycin and nalidixic acid did not inhibit B. pseudomallei at the highest concentration tested $(37 \mathrm{mg} / \mathrm{L}$ and $16 \mathrm{mg} / \mathrm{L}$, respectively) but exhibited activity at these concentrations against B. humptydooensis, B. oklahomensis, B. thailandensis, and B. territorii. Finally, nine of the antibiotics-amoxicillin, ampicillin, cefsulodin, clarithromycin, gentamicin, spectinomycin, streptomycin, puromycin, and paromomycin—did not have activity against the tested $B$. pseudomallei and/or near-neighbor strains, consistent with previous reports of inactivity against various $B$. pseudomallei isolates ${ }^{7,33-36}$ (see Table 1).

These comparative antibiotic susceptibility screens showed that $B$. pseudomallei near neighbors $B$. humptydooensis, $B$. thailandensis, and $B$. territorii have similar antibiotic susceptibility profiles to those of $B$. pseudomallei against key melioidosis therapeutics, as well as other antibiotics (see Table 1, Figure 2).

Susceptibility of $B$. humptydooensis and $B$. territorii to MMV compounds. This study is the first report of antibiotic susceptibilities for $B$. humptydooensis and $B$. territorii. To further evaluate their suitability as models for predicting $B$. pseudomallei drug susceptibility, we also tested their susceptibility to 400 drug-like molecules from the MMV Pathogen Box. ${ }^{39}$ These data allow independent comparison of MMV compound susceptibility of $B$. humptydooensis and $B$. territorii to five previously reported $B$. pseudomallei strains. $^{44}$

We initially tested MMV compounds at $20 \mu \mathrm{M}$ (and identified five compounds with activity against $B$. humptydooensis and $B$. territorii). Four of these compounds-doxycycline, levofloxacin, rifampicin, and MMV688271-agreed with reported activity toward $B$. pseudomallei, ${ }^{44}$ whereas MMV675968 is a newly identified active compound (see Supplemental Table S2 for compound characteristics). Next, we determined the MICs for these compounds against $B$. humptydooensis and $B$. territorii (see Table 2). The activities of doxycycline (MIC 0.5-1 mg/L), levofloxacin (MIC 1-6 mg/L), MMV688271 (MIC 4-8 mg/L), and ceftazidime (MIC 2-4 mg/L) against the near-neighbor strains were within 2-fold of their reported MICs against $B$. pseudomallei (1-3 $\mathrm{mg} / \mathrm{L}, 4-10 \mathrm{mg} / \mathrm{L}, 6-12 \mathrm{mg} / \mathrm{L}$, and 3-4 mg/L, respectively). ${ }^{44}$ Minimal inhibitory concentrations for ceftazidime, doxycycline, and rifampicin determined from the MMV compound screen (Table 2) are in close agreement with MICs from the antibiotic susceptibility screen (Table 1). Principal component analysis of the MICs of these three antibiotics revealed that $B$. humptydooensis

TABLE 2

Minimal inhibitory concentrations of MMV Pathogen Box compounds against $B$. humptydooensis and $B$. territorii strains compared with five B. pseudomallei strains

\begin{tabular}{|c|c|c|c|c|c|c|c|}
\hline \multirow[b]{2}{*}{ Compound } & \multicolumn{2}{|c|}{$\mathrm{MIC}(\mathrm{mg} / \mathrm{L})^{\star}$} & \multicolumn{5}{|c|}{$\mathrm{MIC}(\mathrm{mg} / \mathrm{L}) \dagger$} \\
\hline & $\begin{array}{l}\text { B. humptydooensis } \\
\text { MSMB } 43\end{array}$ & $\begin{array}{l}\text { B. territorii } \\
\text { MSMB } 110\end{array}$ & $\begin{array}{l}\text { B. pseudomallei } \\
\text { K96243 }\end{array}$ & $\begin{array}{l}\text { B. pseudomallei } \\
576\end{array}$ & $\begin{array}{l}\text { B. pseudomallei } \\
\text { NCTC13178 }\end{array}$ & $\begin{array}{l}\text { B. pseudomallei } \\
\text { NCTC13179 }\end{array}$ & $\begin{array}{l}\text { B. pseudomallei } \\
\text { MX2013 }\end{array}$ \\
\hline Ceftazidime $\ddagger$ & $2-4$ & $2-4$ & 4 & 4 & 4 & 6 & 3 \\
\hline Doxycycline & 0.4 & $0.3-1$ & 1 & 2.5 & 3 & 2.5 & 2.5 \\
\hline Levofloxacin & $1-6$ & $1-3$ & 4 & 10 & 6 & 6 & 6 \\
\hline Rifampicin & 13 & $13-26$ & 45 & 18 & 18 & 25 & 18 \\
\hline MMV688271 & $4-8$ & 4-8 & 6 & 12 & 10 & 8 & 12 \\
\hline MMV675968 & $6-12$ & 1 & $>0.72 \S$ & $>0.72 \S$ & $>0.72 \S$ & $>0.72 \S$ & $>0.72 \S$ \\
\hline
\end{tabular}


and $B$. territorii clustered with $B$. pseudomallei strains from this study, and four of the five B. pseudomallei strains were included in the Ross et al. ${ }^{44} \mathrm{MMV}$ compound study (see Supplemental Figure S1).

Newly identified compound MMV67968 showed activity toward B. humptydooensis and B. territorii with MICs (3-12 $\mathrm{mg} / \mathrm{L}$ ) that are less than MICs for rifampicin and only 2-fold higher than MICs for ceftazidime (2-6 mg/L), the "gold-standard" melioidosis therapy (see Table 2). Together, MMV compounds MMV688271 (Ross et al. ${ }^{44}$ study) and MMV67968 (this study) may provide useful information for developing new drugs with activity toward $B$. pseudomallei.

In summary, newly characterized nonpathogenic $B$. humptydooensis and $B$. territorii, and previously described $B$. thailandensis, recapitulate the antibiotic susceptibility of pathogenic $B$. pseudomallei strains, including clinical strains of Mexican, Thai, and Australian origin (MMV compound comparison). ${ }^{44}$ Therefore, each of these strains of nonpathogenic Burkholderia species has potential for use in high throughput in vitro screening of molecules for melioidosis therapy development.

The lower risk-group classification of the near-neighbor species allows expansion of melioidosis research into a wider landscape, where more laboratories have adequate facilities to perform the initial compound discovery. We are hopeful that inclusion of well-characterized and nonpathogenic model organisms in melioidosis research will accelerate the development of new treatment options for this neglected tropical disease.

Received April 6, 2020. Accepted for publication July 6, 2020.

Published online September 21, 2020.

Note: Supplemental figure and tables appear at www.ajtmh.org

Acknowledgments: We would like to thank Alysha Elliot at the Institute for Molecular Bioscience, the University of Queensland, for her critical review of this work; Quentin Kaas at the Institute for Molecular Bioscience, the University of Queensland, for his support with the principal component analysis; Medicines for Malaria Venture for supplying the Pathogen Box compounds; and Vanessa Rigas at the Menzies School of Health Research for her laboratory support.

Financial support: This work was supported by funding from the Australian government (A. S. A. Research Training Program Scholarship), the Australian National Health and Medical Research Council (J. R. W., M. M., and B. J. C. grant numbers 1046812, 1098337, and 1131932, respectively, and the HOT NORTH Initiative; D. J. C., S. T. H., and N. L. grant number 1084965), and the Australian Research Council (Laureate Fellowship to D. J. C. [FL150100146] and Future Fellowship to S. T. H. [FT150100398]).

Authors' addresses: Anna S. Amiss, David J. Craik, and Nicole Lawrence, Institute for Molecular Bioscience, The University of Queensland, Brisbane, Australia, E-mails: a.bundock@imb.uq.edu.au,d.craik@ imb.uq.edu.au, and n.lawrence@uq.edu.au. Jessica R. Webb and Mark Mayo, Global and Tropical Health Division, Menzies School of Health Research, Darwin, Australia, E-mails: jessica.webb@ menzies.edu.au and mark.mayo@menzies.edu.au. Bart J. Currie, Global and Tropical Health Division, Menzies School of Health Research, Darwin, Australia, and Northern Territory Medical Program, Department of Infectious Diseases, Royal Darwin Hospital, Darwin, Australia, E-mail: bart.currie@menzies.edu.au. Sónia Troeira Henriques, Queensland University of Technology, School of Biomedical Sciences, Institute of Healthy and Biomedical Innovation, and Translational Research Institute, Brisbane, Queensland, 4102, Australia, E-mail: sonia.henriques@qut.edu.au.

\section{REFERENCES}

1. Limmathurotsakul $D$ et al., 2016. Predicted global distribution of Burkholderia pseudomallei and burden of melioidosis. Nat Microbiol 1: 15008.
2. Wiersinga WJ, Currie BJ, Peacock SJ, 2012. Melioidosis. NEnglJ Med 367: 1035-1044.

3. Currie BJ, Ward L, Cheng AC, 2010. The epidemiology and clinical spectrum of melioidosis: 540 cases from the 20 year Darwin prospective study. PLoS Negl Trop Dis 4: e900.

4. Limmathurotsakul $D$, Wongratanacheewin $S$, Teerawattanasook $\mathrm{N}$, Wongsuvan G, Chaisuksant S, Chetchotisakd P, Chaowagul W, Day NP, Peacock SJ, 2010. Increasing incidence of human melioidosis in northeast Thailand. Am J Trop Med Hyg 82: 1113-1117.

5. Lipsitz Ret al., 2012. Workshop on treatment of and postexposure prophylaxis for Burkholderia pseudomallei and B. mallei infection, 2010. Emerg Infect Dis 18: e2.

6. Dance D, 2014. Treatment and prophylaxis of melioidosis. Int J Antimicrob Agents 43: 310-318.

7. Thibault FM, Hernandez E, Vidal DR, Girardet M, Cavallo JD, 2004. Antibiotic susceptibility of 65 isolates of Burkholderia pseudomallei and Burkholderia mallei to 35 antimicrobial agents. $J$ Antimicrob Chemother 54: 1134-1138.

8. Leelarasamee A, Bovornkitti S, 1989. Melioidosis: review and update. Rev Infect Dis 11: 413-425.

9. Sarovich DS, Webb JR, Pitman MC, Viberg LT, Mayo M, Baird RW, Robson JM, Currie BJ, Price EP, 2018. Raising the stakes: loss of efflux pump regulation decreases meropenem susceptibility in Burkholderia pseudomallei. Clin Infect Dis 67: 243-250.

10. Jenney AW, Lum G, Fisher DA, Currie BJ, 2001. Antibiotic susceptibility of Burkholderia pseudomallei from tropical northern Australia and implications for therapy of melioidosis. Int $J$ Antimicrob Agents 17: 109-113.

11. Kingsley PV, Arunkumar G, Tipre M, Leader M, Sathiakumar N, 2016. Pitfalls and optimal approaches to diagnose melioidosis. Asian Pac J Trop Med 9: 515-524.

12. Health and Safety Executive, 2013. The Approved List of Biological Agents. London, England: Crown Copyright.

13. The Australia Group, 2014. The Australia Group Common Control List Handbook, Volume II: Biological Weapons-Related Common Control Lists. Available at: https://www.dfat.gov.au/publications/ minisite/theaustraliagroupnet/site/en/controllisthandbooks.html. Accessed October 18, 2019.

14. American Biological Safety Association, 2016. Risk Group Database. Available at: https://my.absa.org/Riskgroups. Accessed October 18, 2019.

15. Centers for Disease Control and Prevention, 2018. Select Agents and Toxins List. Available at: https://www.selectagents.gov/ selectagentsandtoxinslist.html. Accessed October 18, 2019.

16. World Health Organization, 2004. Laboratory Biosafety Manual, 3rd edition. Geneva, Switzerland: WHO.

17. Joint Technical Committee $\mathrm{CH}-026 \mathrm{SiL}, 2010$. Safety in Laboratories. Part 3: Microbiological Safety and Containment. Sydney, Australia: SAI Global Limited, 44-49.

18. Hoffmaster AR et al., 2015. Melioidosis diagnostic workshop, 2013. Emerg Infect Dis 21: e141045.

19. Mishra A, Shamshavali K, Sanathan SR, Rao SB, Talal B, Sravanthi $R, 2017$. Challenges in the diagnosis and treatment of melioidosis: a review. Eur J Pharm Med Res 4: 226-232.

20. West TE, Frevert CW, Liggitt HD, Skerrett SJ, 2008. Inhalation of Burkholderia thailandensis results in lethal necrotizing pneumonia in mice: a surrogate model for pneumonic melioidosis. Trans R Soc Trop Med Hyg 102 (Suppl 1): S119-S126.

21. Kovacs-Simon A, Hemsley CM, Scott AE, Prior JL, Titball RW, 2019. Burkholderia thailandensis strain E555 is a surrogate for the investigation of Burkholderia pseudomallei replication and survival in macrophages. BMC Microbiol 19: 97.

22. Haraga A, West TE, Brittnacher MJ, Skerrett SJ, Miller SI, 2008. Burkholderia thailandensis as a model system for the study of the virulence-associated type III secretion system of Burkholderia pseudomallei. Infect Immun 76: 5402-5411.

23. Propst KL, Mima T, Choi KH, Dow SW, Schweizer HP, 2010. A Burkholderia pseudomallei deltapurM mutant is avirulent in immunocompetent and immunodeficient animals: candidate strain for exclusion from select-agent lists. Infect Immun 78: 3136-3143.

24. Ginther JL, Mayo M, Warrington SD, Kaestli M, Mullins T, Wagner DM, Currie BJ, Tuanyok A, Keim P, 2015. Identification of 
Burkholderia pseudomallei near-neighbor species in the Northern Territory of Australia. PLos NegITrop Dis 9: e0003892.

25. Tuanyok A et al., 2017. Burkholderia humptydooensis sp. nov., a new species related to Burkholderia thailandensis and the fifth member of the Burkholderia pseudomallei complex. Appl Env Microbiol 83: e02802-16.

26. De Smet B et al., 2015. Burkholderia stagnalis sp. nov and Burkholderia territorii sp. nov., two novel Burkholderia cepacia complex species from environmental and human sources. Int $J$ Syst Evol Micr 65: 2265-2271.

27. Limmathurotsakul D et al., 2013. Systematic review and consensus guidelines for environmental sampling of Burkholderia pseudomallei. PLoS Negl Trop Dis 7: e2105.

28. Mayo M, Kaesti M, Harrington G, Cheng AC, Ward L, Karp D, Jolly P, Godoy D, Spratt BG, Currie BJ, 2011. Burkholderia pseudomallei in unchlorinated domestic bore water, tropical northern Australia. Emerg Infect Dis 17: 1283-1285.

29. Currie BJ, Price EP, Mayo M, Kaestli M, Theobald V, Harrington I, Harrington G, Sarovich DS, 2015. Use of whole-genome sequencing to link Burkholderia pseudomallei from air sampling to mediastinal melioidosis, Australia. Emerg Infect Dis 21: 2052-2054.

30. Dutta S, Haq S, Hasan MR, Haq JA, 2017. Antimicrobial susceptibility pattern of clinical isolates of Burkholderia pseudomallei in Bangladesh. BMC Res Notes 10: 299.

31. Inglis TJ, Rodrigues F, Rigby P, Norton R, Currie BJ, 2004. Comparison of the susceptibilities of Burkholderia pseudomallei to meropenem and ceftazidime by conventional and intracellular methods. Antimicrob Agents Chemother 48: 2999-3005.

32. Podin $Y$ et al., 2014. Burkholderia pseudomallei isolates from Sarawak, Malaysian Borneo, are predominantly susceptible to aminoglycosides and macrolides. Antimicrob Agents Chemother 58: 162-166.

33. Fisher MW, Hillegas AB, Nazeeri PL, 1971. Susceptibility in vitro and in vivo of Pseudomonas pseudomallei to rifampin and tetracyclines. Appl Microbiol 22: 13-16.

34. Dance DA, Wuthiekanun V, Chaowagul W, White NJ, 1989. The antimicrobial susceptibility of Pseudomonas pseudomallei. Emergence of resistance in vitro and during treatment. $J$ Antimicrob Chemother 24: 295-309.

35. Vorachit M, Chongtrakool P, Arkomsean S, Boonsong S, 2000. Antimicrobial resistance in Burkholderia pseudomallei. Acta Trop 74: 139-144.

36. Scarff JM et al., 2019. Aminomethyl spectinomycins: a novel antibacterial chemotype for biothreat pathogens. J Antibiot (Tokyo) 72: 693-701.

37. Cockerill F, Wikler M, Alder J, Dudley M, Eliopoulos G, Ferraro M, Hardy D, Hecht D, Hindler J, Patel J, 2012. Methods for dilution antimicrobial susceptibility tests for bacteria that grow aerobically: approved standard. Clin Lab Stand Inst 32: M07-A9.

38. R Core Team, 2020. R: A Language and Environment for Statistical Computing. Vienna, Austria: R Foundation for Statistical Computing. Available at: https://www.R-project.org/.

39. Medicines for Malaria Venture, 2018. Pathogen Box Supporting Information. Available at: https://www.mmv.org/mmv-open/ pathogen-box/pathogen-box-supporting-information. Accessed September 9, 2019.

40. David J, Bell RE, Clark GC, 2015. Mechanisms of disease: hostpathogen interactions between Burkholderia species and lung epithelial cells. Front Cell Infect Microbiol 5: 80.

41. Sawana A, Adeolu M, Gupta RS, 2014. Molecular signatures and phylogenomic analysis of the genus Burkholderia: proposal for division of this genus into the emended genus Burkholderia containing pathogenic organisms and a new genus Paraburkholderia gen. nov. harboring environmental species. Front Genet 5: 429.

42. Paganin P, Tabacchioni S, Chiarini L, 2011. Pathogenicity and biotechnological applications of the genus Burkholderia. Cent Eur J Biol 6: 997-1005.

43. Sahl JW et al., 2016. The effects of signal erosion and core genome reduction on the identification of diagnostic markers. mBio 7: e00846-16.

44. Ross BN, Myers JN, Muruato LA, Tapia D, Torres AG, 2018. Evaluating new compounds to treat Burkholderia pseudomallei infections. Front Cell Infect Microbiol 8: 210. 